\title{
Science et sublime dans la découverte des Alpes
}

\section{Claude Reichler}

\section{Citer ce document / Cite this document :}

Reichler Claude. Science et sublime dans la découverte des Alpes. In: Revue de géographie alpine, tome 82, n³, 1994. pp. 11-29;

doi : 10.3406/rga.1994.3759

http://www.persee.fr/doc/rga_0035-1121_1994_num_82_3_3759

Document généré le 06/06/2016 


\title{
Zusammenfassung
}

Zusammenfassung: Wissenschaft und Erhabenes bei der Entdeckung der Alpen. Diese Studie entspricht einer geschichtlichen Anthropologie der Kultur : sie begegnet hier der Geschichte der Géographie und der Probléme der Entstehung der wissenschaftlichen Kenntnisse und Vorstellungen. Sie hat den Begriff der Erhabenheit zum Ziel, der so oft im letzten Viertel des 18. Jahrhun- derts verwendet wurde. Die hier vorgeschlagene These zeigt, das dieser Begriff die Betrachtungsweisen der Gelehrten, der Malér und der Schriftsteller vereint hat. Zwei Werke werden hier behandelt und verglichen : Die Reisen in die Alpen von H.-B. de Saussure und die alpinen Landschaften des Malers Caspar Wolf. Bei beiden werden die Verbindungen zwischen dem ästhetischen Bewufitsein und der Suché nach einer wissenschaftlichen Objektivierung hinterfragt. Ihre gemeinsame Sorge sich selber als Objekt zu betrachten, sich also bei ihren Observationen auch selbst zu beobachten und somit ebenso die Betrachtung des Subjektes wie auch die Darstellung der bereisten Welt ins Zentrum ihrer Arbeit zu stellen wird hier analysiert. Die beiden Werke sind typisch fur einen Zeitpunkt der Erforschung der Alpen, sie wurden bekannt und in weiten Teilen der Gesellschaft verbreitet. Durch sie haben die zukiinftigen Reisenden und "Touristen" eine neue Wahrnehmung des Hochge- birges erfahren, die sich durch Reise- berichte und Reisefiihrer uber ganz Europa verteilt hat.

\begin{abstract}
Abstract : Science and the sublime in the discovery of the Alps. This study is based on a historical anthropology of culture. Using this approach, it is able to relate to both the history of geography and the pro- blems concerning the construction of scientific knowledge and the imaginary. Its focus is the "sublime", a concept frequently used in the last quarter of the eighteenth century. The proposed thesis shows how this notion unified the approaches of scholars, painters and writers. Two works are discussed and compared : Saussure's Voyages dans les Alpes and the alpine landscape paintings of Caspar Wolf. Questions are raised with regard to the association between aesthetic consciousness and the search for scientific objectifica- tion. The analysis examines the common concern of writer and painter with presenting themselves as the "object", with observing themselves observing and thus with placing the observer's gaze as well as the representations of the real world at the heart of their work. Both examples are characteristic of a time when the Alps were being explored. These mountains became known and knowledge of them was diffused in a fairly extensive social environment. Through these works, travellers and, more recently, tourists obtained a new perception and understanding of high mountain ranges, an understanding which has spread throughout Europe thanks to the contribution of travel narratives and guides.
\end{abstract}

\begin{abstract}
Résumé
Résumé : Cette étude relève d'une anthropologie historique de la culture : c'est par là qu'elle rencontre I'histoire de la géographie et les problèmes de construction des connaissances et des imaginaires scientifiques. Elle a pour objet la notion de sublime, si fréquemment utilisée dans le dernier quart du XVIIle siècle. La thèse proposée ici montre que cette notion unifiait les approches des savants, des peintres et des écrivains. Deux œuvres sont abordées et comparées : les Voyages dans les Alpes d'H.-B. de Saussure et les paysages alpestres du peintre Caspar Wolf. On interroge chez l'un et l'autre les liens de la conscience esthétique et de la recherche d'objecti- vation scientifique. On analyse leur souci commun de se prendre eux- mêmes pour objet, de s'observer observant, et donc de placer au cœur de leur travail le regard du sujet autant que la représentation du monde parcouru. Les deux œuvres sont caractéristiques d'un moment de l'exploration des Alpes; elles furent connues et diffusées dans une aire sociale assez large. A travers elles, les voyageurs et les futurs " touristes » ont appris une perception nouvelle de la haute montage, qui s'est répandue dans toute l'Europe par l'intermédiaire des récits de voyage et des guides.
\end{abstract}

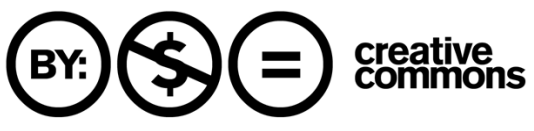




\section{Science ef sublime dans la découverte des Alpes}

Résumé : Cette étude relìve d'une anthropologic historique de la culture : c'est par là qu'elle rencontre l'histoire de la géographic et les problemes de construction des connaissances et des imaginaires scientifiques. Elle a pour objet la notion de sublime, si fréquemment uriliséc dans le dernier quart du XVIII siecle. La thèse proposée ici montre que cette notion unifiait les approches des savants, des peintres et des écrivains. Deux ouvres sont abordées et comparées : les Voyages dans les Alpes d'H.-B. de Saussure et les paysages alpestres du peintre Caspar Wolf. On interroge chez l'un ct l'autre les liens de la conscience esthétique et de la recherche d'objectivation scientifique. On analyse leur souci commun de se prendre cuxmémes pour objet, de s'obscrver observant, et done de placer au cocur de leur travail le regard du sujet autant que la représentation du monde parcouru. I.es deux ceuvres sont caractéristiques d'un moment de l'exploration des Alpes ; elles furent connues et diffusées dans une aire sociale asse\% large. A travers elles, les voyageurs et les fururs " touristes" ont appris une perception nouvelle de la haute montage, qui s'est répandue dans toute l'Europe par l'intermédiaire des récits de voyage et des guides.

Abstract : Science and the sublime in the discovery of the Alps. 'This study is based on a hiscorical anthropology of culture. Using this approach, it is able to relate to both the history of geography and the pro- blems conccrning the construction of scientific knowledge and the imaginary. Its focus is the "sublime", a concept frequently used in the last quarter of the eighteenth century. The proposed thesis shows how this notion unified the approaches of scholars, painters and writers. Two works are discussed and compared : Saussure's Voyages dans les Alpes and the alpine landscape paintings of Caspar Wolf. Questions are raised with regard to the association between aesthetic consciousness and the search for scientific objectification. The analysis examines the common concern of writer and painter with presenting themselves as the "object", with observing themselves observing and thus with placing the observer's gaze as well as the representations of the real world at the heart of their work. Both examples are characteristic of a time when the Alps were being explored. These mountains became known and knowledge of them was diffused in a fairly extensive social environment. Through these works, travellers and, more recently, rourists obtained a new perception and understanding of high mountain ranges, an understanding which has spread throughout lurope thanks to the contribution of travel narratives and guides.

Zusammenfassung: Wissenschaft und Erhabenes bei der Entdeckung der Alpen. Diese Studic entspricht einer geschichtlichen Anthropologie der Kultur : sie begegnet hier der Geschichte der (icographic und der
C. Reichler*

Mots-clefs : Naturalistes, H.B. Saussure, paysage alpin, peinture, sublime, C. Wolf

Keywords : naturalists, H.B. Saussure, alpine landscape, painting, sublime, C. Wolf

Schlüsselwörter : Naturalisten, H.-B. Saussure, alpine Landschaft, Malerei, Erhabenheit, C. Wolf 
Probleme der Entstehung der wissenschaftlichen Kenntnisse und Vorstellungen. Sie hat den Begriff der Erhabenheit zum 7icl, der so oft im letzten Viertel des 18. Jahrhunderts verwendet wurde. Die hier vorgeschlagene These zeigt, daß dieser Begriff die Betrachtungsweisen der Gelehrten, der Maler und der Schriftsteller vereint hat. Zwei Werke werden hier behandelt und verglichen : Die Reisen in die Alpen von H.-B. de Saussure und die alpinen Landschaften des Malers Caspar Wolf. Bei beiden werden die Verbindungen zwischen dem ästhetischen Bewußtsein und der Suche nach einer wissenschaftlichen $\mathrm{Ob}$ jektivierung hinterfragt. Ihre ge- meinsame Sorge sich selber als $\mathrm{Ob}$ jekt zu betrachten, sich also bei ihren Observationen auch sclbst $z u$ beobachten und somit ebenso die Betrachtung des Subjektes wic auch die Darstellung der bercisten Welt ins Zcentrum ihrer Arbeit zu stellen wird hier analysiert. Dic beiden Werke sind typisch für einen Zeitpunkt der Erforschung der Alpen, sic wurden bekannt und in weiten Teilen der Ciesellschaft verbreiter. Durch sie haben dic zukünfrigen Reisenden und "Touristen" einc neue Wahrnehmung des Hochgebirges erfahren, dic sich durch Reiseberichte und Reiseführer über gan\% Europa verteilt hat.
1. Le présent article reprend et développe une conférence donnée au

Centre culturel suisse, à Paris, le 3 décembre 1992.
$\mathrm{E}$ ham Wagner, les Merkwürdige Prospekte aus den SchweizerGebürgen und derselben Beschreibung, traduits presque immédiatement en français sous le titre de Vues remarquables des montagnes de la Suisse'. Il s'agissait d'une entreprise considérable, à la fois scientifique et esthétique, longuement préparée par les excursions sur le terrain accomplies par les principaux acteurs, tous passionnés de montagne : à savoir, outre l'éditeur, le peintre Caspar Wolf et le pasteur Samuel Wyttenbach, auteur des descriptions qui accompagnent les images, féru de géologie, d'hydrologie, de botanique.

Une gravure de Dunker, parue en frontispice, rappelle ces heures mémorables (Photo 1). On y voit, quasiment au centre de l'image, le peintre debout devant son chevalet, palette et pinceaux à la main. Auprès de lui, le petit groupe attentif qui regarde son travail est formé par Wagner et, vue de dos, protégée sous son ombrelle des brûlures du soleil, "Fräulein Müller ", que la description présente comme " une amoureuse ardente des Alpes". A ce groupe très en évidence, qui occupe le premier quart gauche de l'image, fait pendant un autre ensemble de personnages, placé dans le dernier quart de droite, tout au premier plan : on y découvre Wyttenbach notant sur son carnet les indications que lui transmet un paysan. Celui-ci 


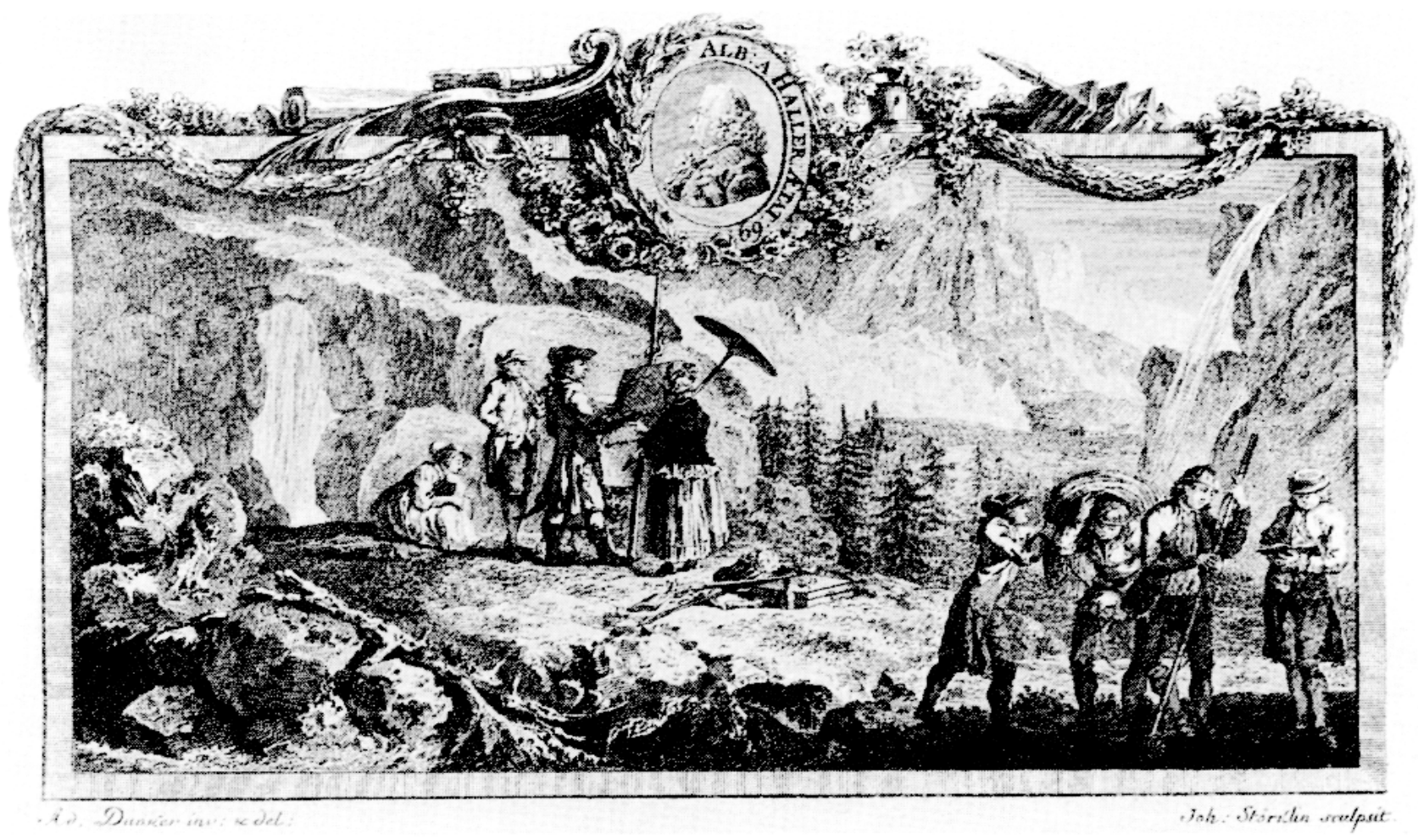

porte un bâton gradué auquel est attachée une corde fort longue, et lourde, puisque l'autre paysan, qui la porte roulée sur son dos, ploie sous la charge. On vient de mesurer la hauteur de la cascade du Staubbach, célèbre par les irisations qu'y produit le soleil matinal. C'est en effet la cascade, à l'entrée de la vallée de Lauterbrunnen, dans l'Oberland, que Wolf est en train de peindre, et dont Wyttenbach prépare la description minutieuse. On voit l'étroite coulisse de la vallée s'enfoncer dans la profondeur, vers la droite, derrière un rideau de sapins, entre les rochers abrupts qui la bordent : paysage déjà bien connu, en ce dernier quart du XVIII" siècle, et promis à devenir un must du voyage en Suisse. Caspar Wolf a donné de très belles gravures de ce site, et quelques peintures sur toile admirables, que ce soit dans un décor hivernal, alors que la cascade gelée s'est figée en un pâle nappé mêlé d'éclats, sur les parois où les vents ont jeté son voile d'eau; ou, l'été, lorsqu'il s'agit de rendre les jeux complexes de la lumière, de l'eau, de la vapeur et de la roche ${ }^{2}$. Placer ce paysage en frontispice des Vues remarquables, c'est montrer Wolf sur son motif, en train de capter une représentation qui est un des emblèmes de son travail, mais aussi qui concentre plusieurs des chocs esthétiques et des problèmes scientifiques proposés aux voyageurs par les Alpes. Le rôle du peintre, que Dunker juge sans doute supérieur à celui de Wyttenbach, est marqué par sa position centrale dans l'orga-
Photo 1

B.A. Dunker, frontispice de l'ouvrage intitulé Merkwürdige Prospekte aus den SchweizerGebürgen und derselben Beschreibung, Berne, 1776-1777, 13,5×21,5
2. La cascade qui se résout en nuée est un défi à la peinture, mais c'est aussi un jeu sur les quatre éléments de l'imagination matérielle: l'eau, l'air, la roche ef le feu du soleil. 
3. le pasteur horrme de science occupe une place apparemment modeste dans le dispositif. Pourtant, la construction de la grande diagonale gauche-

droite, de méme que la lumière qui baigne la partie supérieure droite attire sur lui loeil du spectateur. Fi d'alutre part, il mesure, il écrit, il décrit. i) est géomètre el géographe. Homme du savoir, au service du livre. it a son double prestigieux dans le médaillon.

4. Voir l'édirion de A.

Elschoribroich : Albrecht von Haller, Die Alpen und onderen Cedichte.

Reclom, Siutgart, 1905

p. 17: "Dans les belles vues peintes par Wolf". ajoute Haller en 1777 .

" on voit l'eau dans sa chute se dissouctrce en une nuée." Dans une édition de 1773, il se présentait comme un excursionniste admiratif, dans la position même des personnages de la gravure de Dunker

"J'ai vu l'arc-en-ciel [formé

par la cascade] et suis

demeuré là des heures

durant à observer ce

étonnant phénomène."

5. On consultera la

biographie de Saussure par D.W. Freshfield,

Horace-Bénédict de Saussure, Genève, 1924,

et bien sûr, pour l'histoire de la découverte des

Alpes par les géographes.

Numa Broc Les

Montagnes au siècle des lumières, Paris, 199112 éd.). Voir aussi l'ouvrage nisation de l'image ${ }^{3}$. Dunker s'amuse d'ailleurs à placer la " signature " de Wolf de part et d'autre du centre de la gravure : son fusil posé à terre près de la boite de couleur, et son chien qui nous regarde, à côté du chevalet; l'un et l'autre reviennent en effet très fréquemment dans les toiles de Wolf. Le chien, dont le regard sort de l'image pour y attirer le nôtre, indique, comme par clin d'oil, toute l'importance du regard et du spectacle dans la représentation du paysage alpestre. Le regard du peintre semble commander la perspective, face à la profondeur de la vallée éclaboussée de lumière. Son oeil, allant et venant entre l'objet (la cascade) et l'image en train de naître, semble créer l'espace de la représentation. Les autres personnages ne regardent pas le paysage, mais la toile sur laquelle Wolf le reporte et qui, pour nous, reste dans une ombre opaque. Mais nous voyons ce paysage, et nous voyons le regard du peintre : nous comprenons que le paysage existe dans la mesure où il est représenté, lorsque le travail du peintre lui donne forme. C'est bien là l'ambition des Vues remarquables: donner à voir, c'est-àdire apprendre à voir, former le regard d'un public, toujours plus nombreux, qui découvre les beautés et les mystères d'un espace géographique nouveau.

L'entreprise est placée sous le patronnage de Haller, qui a rédigé une préface et dont le portrait orne le médaillon au sommet de l'image. Rappelons qu'en 1776 Albrecht de Haller (il mourra l'année suivante) est célèbre dans l'Europe entière pour ses travaux dans plusieurs champs scientifiques. Il est médecin et anatomiste de formation. On le surnomme "le Pline de la Suisse " pour ses recherches sur les plantes des Alpes. Directeur des salines de Bex, il s'est intéressé aux formations minérales et au système hydrographique. Mais si sa personnalité vient donner à l'entreprise une caution scientifique, elle l'enrichit aussi du souvenir du poème Les Alpes, publié en allemand par Haller en 1732 , traduit en français en 1750 , et qu'on tient pour une des sources de l'enthousiasme pour la montagne au XVIII' siècle. Le territoire alpin qui, dans ce poème, sert de référence à la description épique, philosophique et pastorale des peuples montagnards, serait précisément constitué, d'après Haller, par les vallées de l'Oberland bernois; la célèbre description de cascade qui occupe les vers 351 à 360 du poème serait le Staubbach. Cette précision est apportée par l'auteur lui-même dans une note ajoutée lors de l'édition de 1748 , et complétée encore lors de la dernière édition parue de son vivant, en $1777^{4}$. L'autorité de Haller rayonne donc de multiples manières sur le travail du 
peintre. Non seulement elle en garantit la justesse et l'objectivité scientifiques, mais elle lui est aussi une caution esthétique. Cette convergence des approches est un phénomène fondamental de la découverte des Alpes. Loin de constituer des compréhensions séparées de leur objet, le savant, le poète et le peintre travaillent à donner accès à un monde unifié.

\section{Voir et savoir}

Un homme, à la fin du siècle, prendra la relève de Haller pour faire connaître les beautés des Alpes et tenter d'expliquer leurs mystères : c'est Horace-Bénédict de Saussure. Il n'est pas sans lien avec notre gravure, puisqu'il est un disciple du grand savant, chez qui il a fait de fréquents séjours, et que Samuel Wyttenbach traduira en allemand ses Voyages dans les Alpes. On sait que Saussure, né en 1740, nommé très tôt professeur de philosophie à l'Académie de Genève, fut possédé par une durable passion pour le Mont-Blanc 5 . Il fut dans ses recherches, et bien que ces sciences ne fussent pas toutes véritablement constituées, géographe autant que géologue, botaniste, minéralogiste, ethnologue et folkloriste des populations de montagne... Dès 1760 , il entreprit des voyages réguliers dans la vallée de Chamonix et le massif du Mont-Blanc. En août 1787, il accomplit la seconde ascension du Mont-Blanc, qui fut perçue comme un événement considérable par toute l'Europe savante et lettrée, grâce notamment à la Relation abrégée d'un voyage à la cime du Mont-Blanc, qu'il publia l'année même, et qu'il reprendra plus en détail dans le tome III de ses Voyages ${ }^{6}$.

L'ouvrage de Saussure, référence de tous les voyageurs curieux et de tous les naturalistes pendant cinquante ans, ne fut pas réédité dans son intégralité au siècle suivant. On en fit paraitre, à partir de 1834, une édition partielle portant le sous-titre de Partie pittoresque 7 . L'ouvrage supprime ou abrège les descriptions spécialisées, tels les nombreux et longs passages sur les roches et les fossiles, les dissertations météorologiques, les considérations théoriques trop développées sur la formation des montagnes : c'est tout le riche savoir naturaliste, certes périmé dès le XIX' siècle, mais pourtant intéressant comme trace d'une recherche patiente, en dialogue avec les conceptions de son temps, qui est en bonne partie écarté. Les éditeurs mettent ainsi en évidence l'exotisme de la montagne, particulièrement dans la perception du paysage, et les confidences que livre l'au- de Philippe Joutard,

L'Invention du Mont-Blanc.

Paris, Gallimard, coll.

"Archives", 1986.

6. Voir H.-B. de Saussure,

Voyages dans les Alpes,

t. I. Neuchâtel, 1779 :

1. II. Genève, 1786; t. III

ef IV. Neuchâtel, 1796.

Slatkine a publié un reprint de cette édition en 1978.

7. Celle-ci fut rééditée au moins 4 fois au cours du $X I X$ siècle; et c'est roujours à partir d'elle, mais en y

joignant la Relation abrégée de 1787, que la Librairie Maspero a fait paraitre, en 1979, le volume intitulé Premières Ascensions au Mont-Blanc (Introduction de Roger Conac). Rodolphe Töpffer, l'auteur des Voyages en zig-zag, écrivit, à

l'occasion de l'édition de

1834, un très bel

hommage à Saussure.

C'est de ce texte sans doute que s'inspire Michelet lorsqu'il écrit, en 1868 : "La gloire de $M$. de Saussure, c'est moins son ascension, et quelques expériences, que son beau voyage imprimé, où il donne sur le Mont-Blanc et les Alpes en général tant de faits intéressants, bien vus, appréciés judicieusement. On sent en lui, ce qui est rare, un homme digne de ce nom, équilibré d'études et de caractère, d'exercice et d'action. "La Montagne, in OEuvres complètes XX, Paris, Flammarion, 1987. p. 103. 
8. " La certitude de jouir pendant plusieurs heures de ce grand spectacle donnait à l'âme une assurance qui redoublait le sentiment de la jouissance ", note-til. (Remarquons la réflexivité.

l'observation de soi qui accompagne la prévision.) teur sur lui-même, ses impressions et ses idées. Ce travail de sélection introduit une séparation entre l'observation scientifique et le récit personnel, et réoriente le texte en répondant à la fois aux attentes des lecteurs romantiques et à l'évolution des récits de voyage.

On peut regretter cette opération, bien qu'elle offre au texte de Saussure un gain de lisibilité évident et utile, et qu'elle le rende accessible aux lecteurs nombreux qu'auraient rebutés les pages d'érudition scientifique. L'édition de 1834 donne à lire Saussure, mais, paradoxalement, occulte un monument de l'histoire du livre de voyage : le caractère encyclopédique, voire hétéroclite, n'est plus perceptible, ni l'unité complexe d'une ouvre qui porte d'un même mouvement une écriture et un savoir, eux-mêmes jamais séparés du sujet qui les élabore et se communique en découvrant un monde.

Le désir de Saussure - parvenir au sommet du Mont-Blanc est un désir de vision totale, panoramique, qui offrirait une maîtrise complète de l'espace, une compréhension sans reste de la structure du monde. Occuper le point où l'intelligibilité est absolue permettrait de voir le monde se déployer comme une carte à multiples lectures : rêve de savant et relève d'une ambition naguère théologique, celle d'occuper la place de Dieu, qui voit tout d'un même regard. Saussure met en scène à plusieurs reprises ce désir de vision totale, de cime en balcon, avec un crescendo dans la complétude. Le lecteur approche peu à peu du but poursuivi, comme si toute l'œuvre était aimantée par ce moment de perfection. Si longs et touffus qu'ils paraissent, les Voyages dans les Alpes obéissent à une structure narrative simple, celle de la quête du point idéal, qui est aussi révélation d'une connaissance progressivement acquise.

Voici un passage paru dans le volume II, en 1779. Nous sommes sur le Cramont, dont Saussure a fait l'ascension pour la seconde fois l'année précédente. Les conditions météorologiques sont idéales ${ }^{8}$. Saussure prend des mesures angulaires, analyse l'organisation des sommets et des vallées tout en admirant la beauté du point de vue. Puis ses réflexions s'élargissent, passent de la vision actuelle de la chaîne du Mont-Blanc à la vision théorique de la formation des Alpes, dans un véritable morceau de bravoure, qu'il faut citer en entier : 
Je voyais cette chaîne composée de feuillets que l'on pouvait considérer comme des couches; je voyais ces couches verticales dans le centre de cette chaîne et celles des secondaires presque verticales dans le point de contact avec elles, le devenir moins à de plus grandes distances, et s'approcher peu à peu de la situation horizontale, à mesure qu'elle s'éloignait de leur point d'appui : je voyais ainsi les nuances entre les primitives et les secondaires, que j'avais déjà observées dans la matière dont elles sont composées, s'étendre aussi à la forme et à la situation de leurs couches, puisque toutes les sommités secondaires que $j$ 'avais là sous les yeux se terminaient en lames pyramidales aiguës et tranchantes, tout comme le Mont-Blanc et les montagnes primitives de la chaîne. Je conclus de tous ces rapports, que puisque les montagnes secondaires avaient été formées dans le sein des eaux, il fallait que les primitives eussent aussi la même origine. Retraçant alors dans ma tête la suite des grandes révolutions qu'a subies notre globe, je vis la mer, couvrant jadis toute la surface du globe, former par des dépôts et des cristallisations successives, d'abord les montagnes primitives, puis les secondaires ; je vis ces matières s'arranger horizontalement par couches concentriques; et ensuite le feu, ou d'autres fluides élastiques renfermés dans l'intérieur du globe, soulever et rompre cette écorce, et faire sortir ainsi la partie intérieure et primitive de cette même écorce, tandis que ses parties extérieures ou secondaires demeuraient appuyées contre les couches intérieures. Je vis ensuite les eaux se précipiter dans des gouffres crevés et vidés par l'explosion des fluides élastiques. Et ces eaux, en courant à ces gouffres, entraîner à de grandes distances ces blocs énormes que nous trouvons épars dans nos plaines. Je vis enfin après la retraite des eaux les germes des plantes et des animaux, fécondés par l'air nouvellement produit, commencer à se développer, et sur la terre abandonnée par les eaux, et dans les eaux mêmes, qui s'arrêtèrent dans les cavités de la surface?.

Cette grande vision de la formation des montagnes est le récit d'une Genèse scientifique. Les théories de l'époque, neptunisme et plutonisme, s'y mêlent en une synthèse originale, qui culmine dans une puissante évocation des matières élémentaires, qui toutes concourent à la création de la vie. Après une première séquence contemplative, où le monde observé se donne à voir dans la clarté des rapports établis entre ses parties, par une analyse qu'on pourrait dire géomorphologique (la forme et la situation de leurs couches) et synchronique, vient le récit historique (la suite des grandes révolutions) où tous les éléments acquièrent un dynamisme digne de la grandeur et de la violence d'un monde sauvage en gestation. On aura remarqué que le verbe voir, répété trois fois dans la phase de contemplation actuelle, effective (je voyais), est utilisé à nouveau à quatre reprises dans la séquence imaginaire (retraçant alors dans ma tête
9. Voyages dans les Alpes, 1. 11, chap. XXXIV. p. 339-340 (éd. 1786). J'ai adapté l'orthographe aux usages actuels. Les éditions romantiques comportent ce passage; voir aussi l'éd. Maspéro, p. 151-152. Numa Broc commente certains aspects de ce texte dans son chapitre sur l'origine des montagnes. 
(...], je vis). La vision de l'observateur, qui segmente, classe, relie, se transforme en une sorte d'hallucination scientifique, admirable du point de vue littéraire et rhétorique. Saussure fait surgir sous les yeux de son lecteur, en un spectacle accéléré, le chaos fertile d'où sort la vie, la dévastation qui devient ordre et système. Ecrivain formé à la rhétorique classique, c'est la figure de l'hypotypose, apte à susciter l'émotion du lecteur, qu'il met ici en ouvre : elle rend présents les objets absents, elle fait revenir le passé, comme en un théâtre où des fantômes prendraient vie devant les spectateurs. Cette violence primitive et ses effets sur l'imaginaire sont souvent évoqués par les voyageurs des Alpes : ils ont le sentiment de contempler l'origine du monde, d'être devant une violence chaotique surgie sous leur regard, au moment de la séparation des éléments. Voyez Hugo, en 1839, au sommet du Rigi

Ces montagnes sont des vagues en effet, mais des vagues géantes. [...] On croirait voir un océan monstrueux figé au milieu d'une tempête par le souffle de Jéhovah. Un rêve épouvantable, c'est la pensée de ce que deviendraient l'horizon et l'esprit de l'homme si ces énormes ondes se remettaient tout à coup en mouvement. ${ }^{10}$

10. Paru dans le Rhin en 1845 ; cité ici d'après Victor Hugo, Voyages en Suisse, L'Age d'homme, Lausanne, 1982.

11. La puissance de l'écriture de Saussure a été admirée dès le début: Monsieur de Saussure, écrit ainsi Kant dans le chapitre sur le sublime de

la Critique de la faculté de juger (1790), * cet homme excellent [qui] ressentait des sensations qui transportent l'ârne et les communiquait aux lecteurs de ses voyages. "

\section{Un nocturne sublime}

Ces grandes orchestrations, qui réalisent euphoriquement la compréhension absolue que recherche Saussure, dans un mouvement où la science, l'imaginaire et la dramaturgie des idées concourent à captiver le lecteur, ont été reçues comme un témoignage particulièrement fort du sublime de la montagne. Des générations de lecteurs et de voyageurs y ont appris l'émotion et l'exaltation devant le spectacle de la nature ${ }^{11}$. Elles ont un contrepoint sombre dans le texte des Voyages, comme si le sujet, exalté par la force de sa vision, était parfois saisi d'angoisse devant la totalité qu'il entrevoit. La position de maîtrise disparaît, la compréhension se défait ; le monde alors devient insaisissable et se perd dans l'infini. Voici à nouveau une page des Voyages, tirée des excursions des années 80 sur les pentes du Mont-Blanc. Là encore, Saussure s'est d'abord concentré sur des observations scientifiques, qu'il n'a d'ailleurs pas pu réaliser, faute d'un appareil adéquat :

Mais la beauté de la soirée et la magnificence du spectacle que présenta le coucher du soleil depuis mon observatoire, vint me consoler 
de ce contretemps. I a vapeur du soir, qui comme une gaze légère, tempérait l'éclat du soleil, et cachait à demi l'immense étendue que nous avions sous nos yeux, formait une ceinture du plus beau pourpre qui embrassait toute la partic centrale de l'horizon ; tandis qu'au levant les neiges des baes du Mont-Blanc colorées par cette lumière, présentaient le plus grand et le plus singulier spectacle. A mesure que la vapeur descendait en se condensant, cette ceinture devenait étroite et plus colorée; enfin clle parut d'un rouge de sang, et dnas le même instant, de petits nuages qui s'élevaient au-dessus de ce cordon, lançaient une lumière d'une si grande vivacité qu'ils semblaient des astres ou des météores embrasés. Je retournai là lorsque la nuit fut entièrement close ; le ciel était alors parfaitement pur et sans nuages, la vapeur ne se voyait plus que dans le fond des vallées : les étoiles brillantes, mais dépouillées de toute espèce de scintillation, répandaient sur les sommités des montagnes une lueur extrêmement faible et pâle, mais qui suffisait pourtant à faire distinguer les masses et les distances. Le repos et le profond silence qui régnaient dans cette vaste étendue, agrandie encore par l'imagination, m'inspiraient une sorte de terreur ; il me semblait que j'avais survécu scul à l'univers, et que je voyais son cadavre étendu sous mes pieds. Quelques tristes que soient les idées de ee genre, elles ont une sorte d'attrait auquel on a de la peine à résister. Je tournais plus fréquemment mes regards vers cette obscure solitude, que du côté du Mont-Blanc, dont les neiges brillantes et comme phosphoriques donnaient encore l'idée du mouvement et de la vie ${ }^{12}$.

I.à encore, le spectacle est en deux temps. Le premier, tout de brillance et de couleurs, se présente comme une sorte de fête de la lumière, qui apparaît sous tous ses aspects, tamiscee, réfléchie ou concentrée. La description insiste sur la " picturalité " du spectacle : elle différencie les nuances de la couleur, la qualité des matières traversées ou réfractantes (gaze légère, neiges, vapeur, nuages), et elle fait référence à une catégorie répertoriée du canon classique, le "grand ", qui est au-delà du beau et donne à tout ce passage une teinte épique. Les expressions hyperboliques, les superlatifs, les termes venus de la théorie esthétique (beauté, magnificence, grand et singulier spectacle), et jusqu'à la comparaison avec les corps célestes, astres et météores, confirment ce registre. Mais là encore, Saussure reste soucieux d'exactitude scientifique, et sa description constitue aussi l'étude d'un phénomène météorologique en haute altitude, qu'on pourrait traduire en termes de pression, de densité et d'angle de réfraction. La position dominante des observateurs leur en permet la plus complète jouissance et la plus fine perception.

I a seconde phase, nocturne, solitaire et angoissée, sera tout opposée à celle-ci. Le monde, d'où s'est absentée la lumière, est
12. Tome II, chap. III p. 501562 . Ce passage

on le comprend, sera lui

oussi repris par es

éditeurs romanticaues

it igure cuse p 178.179

de l'éd. Masperers. 
13. On pourrait accumuler les exemples. Voici le premier premier belvédère des Voyages, au sommet de la Dôle: "Pour jouir de ce spectacle dans tout son éclat, il faudrait le voir comme le hasard me l'offrit un jour. Un nuage épais couvrait le lac, les collines qui le bordent, et

même toutes les basses montagnes. Le sommet de

la Dóle et les hautes

Alpes, étaient les seules

cimes qui élevassent leurs têtes au-dessus de cet immense voile: un soleil brillant illuminait toute la surface de ce nuage: et les Alpes éclairées par les rayons directs du soleil, et par la lumière que ce nuage réverbérait sur elles, paraissaient avec le plus grand éclat, et se voyaient à des distances prodigieuses. Mais cette situation avait quelque chose d'étrange et de terrible : il me semblait que j'étais seul sur un rocher au milieu d'une mer agitée, à une grande distance d'un continent bordé par un long récif de rochers inaccessibles. "

(Tome I, chap. XV,

p. 289) Le lien entre mer et montagne deviendra topique; voir les pages qu'Alain Corbin consacre à cette proximité dans les Territoires du vide.

L'Occident et le désir du rivage 1750-7840. Paris, Aubier, 1988.

14. Une recherche

biographique aurait chance de retrouver trace de leur possible rencontre. Les toiles de Wolf étaient connues dans ces années. menacé de disparition. Le sujet, comme envahi par la pâleur funèbre émanée des choses, dépasse toute idée esthétique et toute tentative d'analyse objective, pour accéder à un sentiment quasi religieux, placé sous le règne de l'imagination, mais aussi d'une ambiguiité psychologique que Saussure note avec insistance : il éprouve, dit-il, une sorte de terreur, qu'il ressent en même temps comme une sorte d'attrait quasi irrésistible. L'observation du monde a fait place à l'observation de soi, ou plus exactement à l'observation du retentissement en soi du spectacle du monde. La vision de l'obscure solitude, l'idée de l'anéantissement de l'univers s'éprouvent dans le sujet comme intuition de sa finitude et de son isolement.

Saussure, qui s'entend remarquablement à rédiger une description géographique de l'espace alpin, sait aussi mettre en scène une dramaturgie de la perception et de la connaissance, parce qu'il est attentif à tourner vers son propre moi l'acuité de son analyse, qu'il se fait l'observateur du sujet en train d'observer ${ }^{13}$. Les témoignages de ce regard dédoublé, de cette capacité de se prendre soi-même pour objet sont fréquents dans les Voyages, et ne concernent pas que l'aspect psychologique des perceptions en haute altitude. Saussure note aussi la qualité de sa respiration, compte ses pulsations, est attentif à la fatigue, à l'appétit, au sommeil, comme à autant de symptômes des modifications physiologiques induites par la montagne. Pourtant, c'est l'observation psychologique qui est la plus intéressante et la plus significative, parce qu'elle révèle en Saussure un relais important de l'histoire de la sensibilité ou, pour employer une expression qui me paraît préférable, de l'histoire de la culture. Ce retour du sujet sur sa perception et sur son émotion, fait apparaître chez Saussure une mise en ouvre complexe, et remarquablement unifiée, des conceptions du sublime élaborées durant tout le XVIII siècle, et répercutées encore au siècle suivant. Avant de le montrer, je voudrais analyser quelques aspects de l'œuvre de Wolf, qui peuvent être tenus pour les correspondants picturaux des descriptions saussuriennes.

\section{Le peintre dans le tableau}

Rapprocher Saussure et Wolf (la partie de l'œuvre de Wolf qui a trait à la montagne) apporte un éclairage fécond sur la représentation des Alpes à la fin du XVIII ${ }^{c}$ siècle. Tout invite à ce rapprochement. L'activité de peintre des Alpes de Wolf, qui a 
duré esentiellement de 1773 à 1779 , est contemporaine de la préparation et de la parution du premier tome des Voyages ${ }^{14}$. Du point de vue de l'analyse historique de la culture, la congruence est frappante, dans les thèmes forts des ouvres comme dans la capacité commune d'unifier l'approche esthétique et les préoccupations scientifiques. Wolf en effet est un artiste très réfléchi, informé des problèmes scientifiques de l'exploration des Alpes; son intérêt pour la géologie et la topographie est partout visible dans ses travaux, de même que sa curiosité pour les formations glacières, dont la théorie passionnait alors le monde savant ${ }^{15}$. De plus Wolf, qui avait été à Paris l'élève de Loutherbourg et l'admirateur de Joseph Vernet (le peintre des effets de lumière et des marines), a transporté dans les paysages de haute montagne le sublime des tempêtes et des naufrages. Il fut, dit un voyageur allemand, " le peintre des beautés sublimes, tendres et effrayantes, de la Suisse "16. C'est cela qui nous retiendra ici, non pas seulement pour les contenus " sublimes", les objets ou aspects du monde qui reçoivent une telle qualification, mais aussi pour la présence dans son œuvre de cette caractéristique à la fois épistémologique et esthétique découverte déjà chez Saussure : la duplication du sujet, à la fois peignant et peint, observateur et observé.

Wolf a peint à plusieurs reprises un site qu'il affectionnait, les grottes de Saint Béat, au-dessus du lac de Thoune (Photo 2). Le lieu a une histoire, que le peintre laisse deviner par allusions. Vastes et traversées par un ruisseau qui en sort pour se perdre dans la pente boisée, vers le lac, elles ont été probablement habitées à l'époque préhistorique. Un dragon, disait la légende médiévale, s'y tenait autrefois et dévorait les imprudents : des ossements trouvés dans le sol en témoignent... Beatus, le saint ermite, aurait chassé le monstre et se serait installé dans la grotte, devenue, après sa mort, un lieu de pèlerinage. On y avait au Moyen Age édifié un sanctuaire, que la Réforme a détruit, et dont les ruines s'aperçoivent dans les toiles de Wolf. Au XVIII ${ }^{c}$ siècle, ce sont les voyageurs qui se rendent aux grottes, pour la beauté du site et la pointe d'angoisse que font naître leurs sombres profondeurs, contrastant avec le paisible belvédère sur le lac et les montagnes. Dans une toile, Wolf montre une société de voyageurs en train de visiter les lieux, par un jour d'été éclaboussé de lumière ${ }^{17}$. Un serviteur ramasse du bois, un autre s'active à faire le feu, dont la fumée monte en volutes; des enfants jouent ; une belle dame en longue robe, sous son ombrelle, regarde le paysage en bavardant ; un homme
Les voyageurs passant par Berne les voyaient chez l'éditeur Wagrer : Coxe ef Ramond de Carbonnières les men:ionnent. Quant dux recherches de Saussure. elles furent 'objet d'un intérêt public avant la parution de son premier volume, el elles étaient discutées dans le milieu de savanis el d'excursionisies passionnés par les Alpes. Haller lui-même, outant que Wyttenbach, a pu constituer un lien entre les cuvres, sirion entre les personnes.

15. Il connait l'ouvrage de son compatriote G.S. Gruner, Die Eisgebirge des Schweizerlandes. paru en 3 vol. à Berne de 1760 c 1762 lla traduction française paraitra en 1770 à Paris, sous le titre, inspiré par Buffon, de Histoire nafurelle des glacières de la Suissel. Ce livre, fort lu du public de langue allemande, compte autant par son apport dans I'histoire des sciences naturelles, que par la splendide violence de ses descriptions.

16. K.G. Kümer, Briefe eines Sachsen aus der Schweiz..., Leipzig, 1785. 17. La grotte de Saint-Béat avec une société de voyageurs (1776), huile sur toile, $54 \times 82$,

Aargauische

Kunstsommlung, Aarau.

Deux expositions centrées sur l'ceuvre alpestre de Wolf ont eu lieu récemment, toutes deux accompagnées d'un catalogue: Caspar Wolf. 


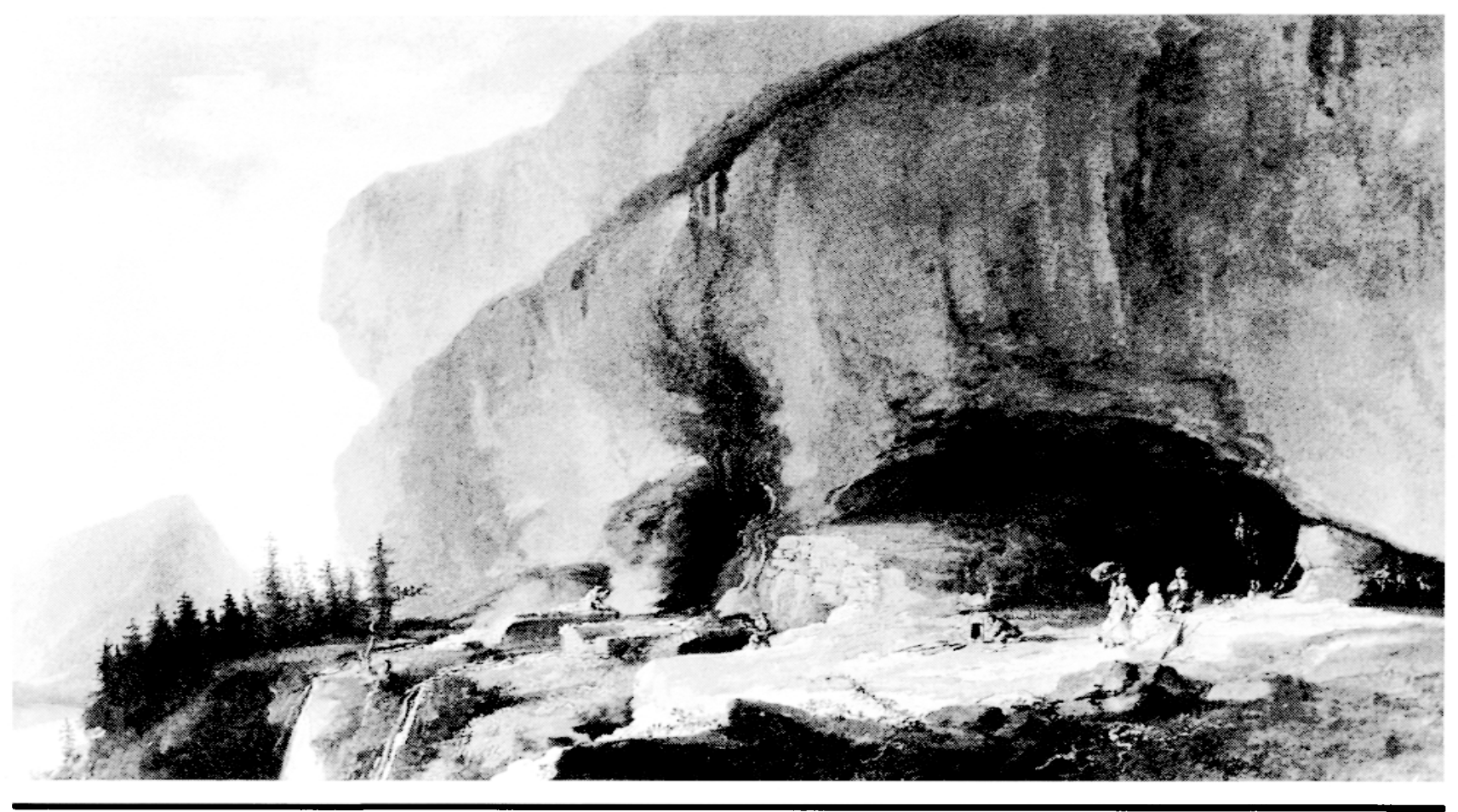

Photo 2:

C. Wolf, Vue de la grotte de Saint Beat près du lac de Thoune, 1776, huile sur toile, $54 \times 82$

Landschatt im Vorfeld der Romantik, au Kunstmuseum de Bôle, 1980; et

l'Hommage à Caspar Wolf conçu par Beat Wismer à l'Aargauer Kunsthaus d'Aarau en 1991. On doil à Willi Rueber (Caspar Wolf 11735.17831. Sein Leben und sein Werk, Zurich,

1979) la redécouverte de

l'oeuvre alpestre de cel artisie, laissée dans

'ombre pendant près de deux siecles. Voir aussi Madeleine Pinault, Le peintre et l'histoire naturelle, Paris, 1990 pénètre dans la grotte, dont l'une des entrées s'ouvre comme une voûte. Wolf représente avec soin les deux bancs massifs superposés qui forment la falaise, le surplomb au-dessus de la voûte, la couleur brune et grise de la roche avec ses coulées d'humidité, le ruisseau, l'esplanade et la forêt de coniferes. Sa description constitue presque une analyse géologique ; elle permet de reconnaître la nature calcaire du rocher et les modifications qu'y apporte l'eau par infiltration ou par ruissellement. Tout à gauche du tableau, à l'écart, on aperçoit le peintre en train de travailler. Il est assis sur une pierre devant une autre entrée qu'on voit latéralement. Un lierre, collé contre la falaise, lance très haut ses ramifications serpentines.

Une autre toile, de la même année 1776, montre ce lieu du peintre, comme si un détail du tableau précédent avait été agrandi ${ }^{18}$. Pourtant, cette comparaison est fausse : la disposition spatiale n'est pas la même, l'entrée de la grotte est cette fois-ci frontale, vaste gueule noire qui occupe tout le centre du tableau et aspire le regard. Le ruisseau s'échappe dans la lumière. Le lierre, sur la droite, se tord étrangement. Le peintre qui le dessine lui fait face, tout à gauche; nous le voyons de côté, petite silhouette assise, un carton sur les genoux. Dans cette toile, Wolf dépasse - après nous les avoir montrés - l'anecdote et le pittoresque de la représentation alpine. Plus d'aimable société d'ex- 
cursionnistes, plus de ciel clair ni de plan d'eau, plus d'allusion à l'idylle champêtre, dont les bords du lac de Thoune sont une localisation déjà traditionnelle. Plus de description de ces formations rocheuses que son art nous faisait voir comme des ruines, érodées et mangées par les eaux et les vents, pas mieux préservées des ravages du temps que les pierres du sanctuaire médiéval dévasté. Au lieu de cela, le peintre se fixe sur deux motifs. Le premier, apparemment secondaire : le lierre, végétal soudé au minéral, traité comme un objet magique, presque animé dans sa torsion ; symbole d'éternité et de fidélité, le lierre participe de la thématique du temps - géologique, archéologique, social qui imprègne les grottes de Saint-Béat. Le second motif, c'est l'antre, bien sûr, le mystère infernal de cette invitation inquiétante d'où sort la source vive. Au-delà du monde socialisé et connaissable des Alpes, Wolf nous fait pressentir, dans cette méditation tellurique à laquelle invite le trou obscur, la présence proche d'un monde originaire toujours inconnu.

"L'homme ", dit Baldine Saint-Girons, " trouve son aliment dans l'obscurité, alors même qu'il s'enchante de clarté ; il aspire au beau, mais il est arrêté par le sublime "19. Si notre regard ne peut pénétrer dans la profondeur de la grotte, nous pouvons voir ce que dessine le peintre sur son carton. Celui-ci existe en effet, ou du moins une ceuvre de petit format censée être tel ${ }^{20}$. Le caractère d'ébauche, la présence dynamique de la pâte et de la touche, la tension entre surface et volume, en font une œuvre étonnamment moderne, où la représentation semble céder le pas à la matérialité picturale. A la vue, au remarquable, au social, à la curiosité scientifique et historique, à toutes les dimensions " éclairées ", objets conventionnels de la peinture, Wolf substitue une relation immédiate et intime avec la matière rocheuse autant qu'avec l'acte de peindre. Alors que les deux œuvres précédentes répondaient à des catégories esthétiques connues et témoignaient d'une réflexivité maîtrisée, " objective ", celle-ci relève d'une représentation en train de se faire, ou hésitant à se faire, prête à sombrer dans la seule matière. Ne s'apparente-t-elle pas au sentiment de l'infini, que les romantiques invoqueront lorsque le sujet se perd dans sa vision et s'enfonce dans la matière du monde, ne faisant plus qu'un avec elle? Les Alpes seront au XIX' siècle un lieu privilégié où éprouver ce sentiment de l'infini. Le jeune Hegel en a une intuition fort précise et remarquablement formulée lors de son voyage dans l'Oberland, en 1796, devant la cascade de Reichenbach :
18. Entrée ouest de la grolte de Saint Béat avec le lierre (1770), huile sur toile $54 \times 70$.

Aargauische

Kunstsammlung, Aarau.

19. Avantpropos à

l'édition française de

Burke, Recherches

philosophiques sur

l'origine de nos idées de sublime et de beau, Paris, 1973.

20. Il s'ogit d'une huile sur carton, $23,8 \times 38,6$, qui porte le même titre et se trouve aussi au musée d'Aarau. Wolf avait coutume d'emmener avec lui, dans ses excursions, des cartons de ce format sur lesquels it traçait ses esquisses. 
Photo 3

C. Wolf, Orage sur le glacier inférieur de Grindelwald, 1774-

1775, huile sur toile,

$54 \times 76$

Photo 4

C. Wolf, Le glacier du Rhône vu de la vallée près de Gletsch, 1778, huile sur toile, $54 \times 76$
21. Cité d'après G.W.F. Hegel, Journal d'un voyage dans les Alpes bernoises, trad. de R.

Legros et F. Verstraeten, éd. J. Millon, 1988.

Hegel, dans tout son

voyage, critique vivement

les descriptions des voyageurs et les

impressions sublimes. Il est

tout aussi insensible à la compréhension scientifique des montagnes.

22. Huile sur toile,

$54 \times 76$, Aargauische

Kunstsammlung. Aarau.

On sait que la deuxième moitié du XVIII ${ }^{e}$ siècle est une période d'avancée glacière qui a beaucoup impressionné les

observateurs. Le tableau de Wolf constitue un document quasi

photographique sur l'état du glacier à son époque et témoigne aussi de

l'inquiétude plus générale des populations de montagne.
Un mince filet d'eau surgit d'une étroite fente dans le rocher, puis retombe verticalement en des flots plus importants; des flots qui entrấnent continuellement le regard du spectateur, mais qu'il ne peut jamais fixer ni suivre, car leur image, leur figure se dissout à chaque instant ; chaque flot est à tout moment chassé par un autre et, dans cette cascade, le spectateur voit éternellement la même image, et simultanément il voit que ce n'est jamais la même 21.

Mais Wolf n'est pas un romantique ; si son œuvre approche une intuition de l'infini, c'est plutôt comme une limite en-deçà de laquelle elle se tient, un état-limite dont elle se préserve plutôt qu'elle ne s'y livre. La fusion du sujet et de l'objet menacerait de dissoudre le point de vue scientifique et la jouissance sublime; Wolf cherche, lui, à les construire par son travail de peinture. C'est pourquoi ses tableaux montrent si fréquemment le peintre sur le motif, certes réduit à peu devant l'immensité de la nature alpestre, mais pourtant présent et témoignant d'une vive conscience de son rôle. Etudions-le sur de nouveaux exemples.

Wolf a dessiné plusieurs des grands glaciers des Alpes suisses pour les collections de Wagner auxquelles il a collaboré. Il en a peint un certain nombre sur toile : le glacier de l'Aar, celui de Grindelwald (dont une version sous l'orage, et striée d'éclairs, photo 3), celui du Rhône. Le désir du spectaculaire, la représentation du "grand théâtre de la nature " n'oblitèrent jamais le souci de l'exactitude topographique et géologique. Son information sur la structure des glaciers et la position des sommets adjacents répond aux connaissances les plus avancées de son temps, et la mise en place topologique précise vient encore renforcer l'impression d'une nature surhumaine, voire inhumaine, auprès de laquelle les hommes sont réduits à l'état de points colorés ou de silhouettes à peine visibles. Ainsi en va-t-il dans la toile peinte en 1778, montrant le glacier du Rhône vu de Gletsch (Photo 4) 22 . L'immense coulée de glace qui occupe tout l'avant-plan, poussant devant elle la moraine frontale récente, paraît terrifiante, prête à tout envahir, comme une vague géante momentanément figée. Tout à gauche du tableau, minuscule, découpé sur le fond d'une tache de lumière, le peintre assis sur un pliant dessine, accompagné d'un serviteur et de son chien. Sa position latérale et basse, très différente de la position théorique du spectateur, qui est centrée et en légère contreplongée, ajoute à l'impression de menace, au sentiment de solitude. Le peintre sur le motif apparaît ici comme ce " sujet 

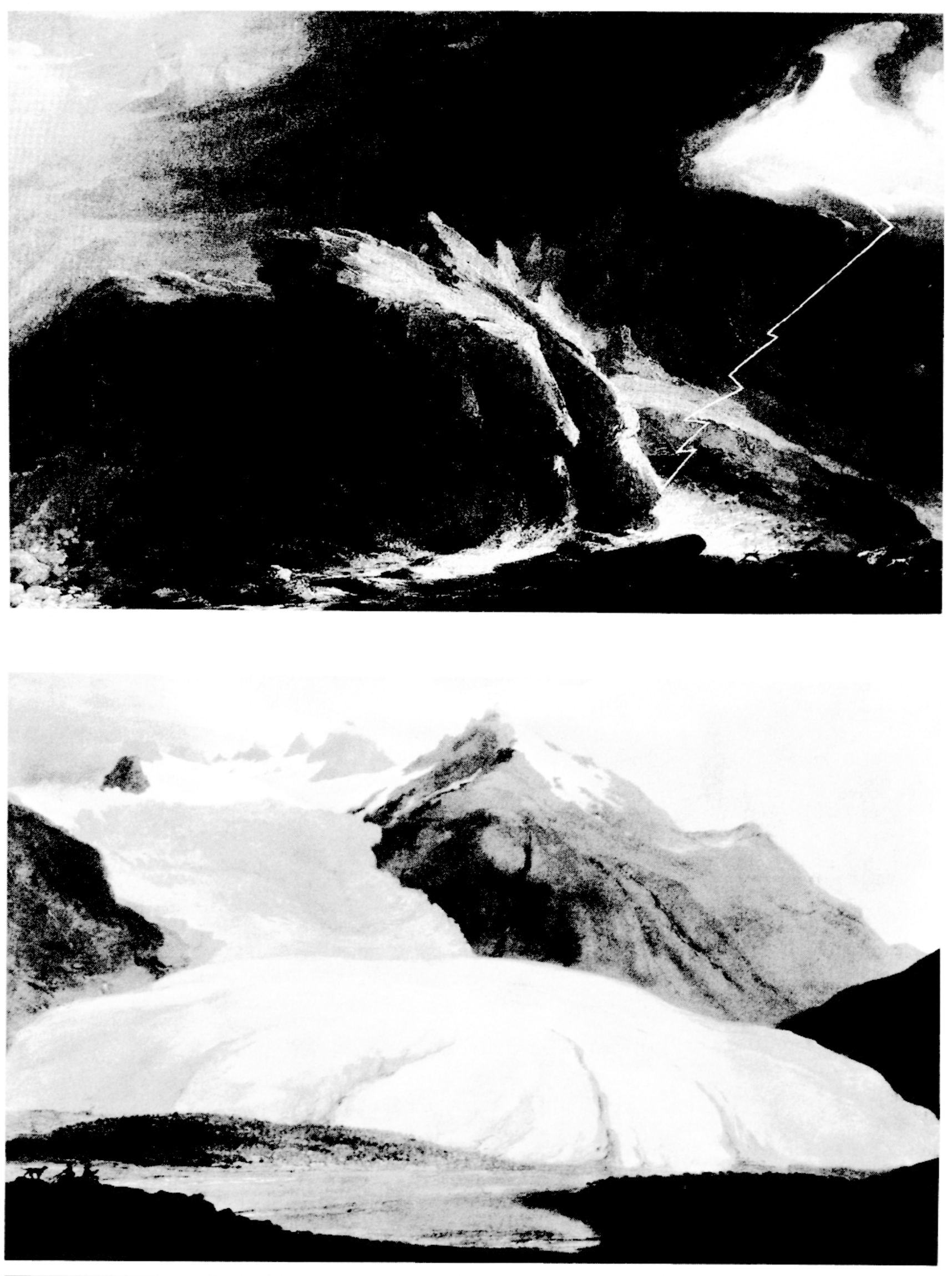
sublime " dont Saussure note les impressions, confronté à un monde qui pourrait l'écraser et qui le nie, et dont pourtant il évite la menace puisqu'il en jouit esthétiquement. Autant que de l'objet naturel, le sublime se dégage de cette mise en scène de soi en train de peindre, de cette capacité de représenter sa propre fragilité comme une jouissance supplémentaire.

Dans une toile datant probablement de $1773^{23}$, qui est un paysage composé ("Ideallandschaft ") juxtaposant des sites parfois reconnaissables et montrant un ensemble grandiose de roches, de cascades, de cimes, de nuées dramatiques et de glaciers suspendus, en un mot tout l'appareil sublime, Wolf se livre à une sorte de description géomorphologique. Il indique à la fois l'idée d'un surgissement primitif des masses dressées vers le ciel, et l'érosion qui les dévaste. Il peint les roches fracassées par éboulement, les modifications apportées par la pression des glaces, la fonction du ruissellement des eaux, qui découvre la structure des plaques schisteuses. Mais cette minutie inspirée par la science ne l'empêche pas de poursuivre aussi une visée artistique. Il se représente lui-même à l'avant-plan, assis contre un rocher et dessinant, chien et fusil fidèlement placés à ses côtés. Un peu en arrière, sur la droite, deux personnages se reposent près d'un feu. Que dessine le peintre ? Certes pas le paysage que nous voyons, puisque celui-ci est derrière son dos. Et pas autre chose non plus, puisque le genre du paysage "composé " ou "idéal " ne peut que varier les éléments du vocabulaire formel attesté par le tableau. Le peintre est ici au centre du monde qu'il transpose dans sa peinture ; et en même temps le paysage actualise les formes que travaille l'artiste. Il y a là comme un double solipsisme : le monde accomplit l'œuvre de l'artiste qui le représente. L'un et l'autre sont sujets de représentation, dans les deux sens de l'expression, comme le laissait voir la gravure de Dunker que nous avons analysée au début de cette étude.

23. Paysage de hautemontagne avec des alpinistes au repos et le peintre, huile sur toile, $52 \times 65$, collection privée, Berne. Je remercie Jörg Winistörfer pour les informations géologiques qu'il m'a aimablement communiquées.

\section{Un concept unificateur}

Le rationalisme cartésien avait pu faire bon ménage avec les idées venues de la Bible (par exemple pour la chronologie de la Terre ou la théorie dite "diluvienne ", encore soutenue au début du XVIII' siècle) ou avec les traditions folkloriques. Les livres de Scheuchzer, le grand naturaliste admiré durant toute la première moitié du XVIII ${ }^{\circ}$ siècle, sont remplis de références 
bibliques et de dragons ${ }^{24}$. Un ouvrage de large diffusion comme L'Etat et les délices de la Suisse, qui compile abondamment Scheuchzer, comporte dans sa première édition de 1730 un long chapitre sur les dragons et des pages sur le déluge. Les nouvelles conceptions scientifiques éclairées, c'est-à-dire critiques, la physique newtonienne, la perspective historique tracée par Buffon, rendront caduques ces survivances du merveilleux et discréditeront les connaissances venues de la tradition populaire ${ }^{25}$. D'autre part, la vision esthétique, formée par le goût classique du paysage à la Poussin ou à la Claude Lorrain, et prisonnière du " géométrisme " des jardins français, échoue à juger des sentiments qu'inspire une nature non ordonnée, irréductible aux canons de la symétrie et de la proportion. Un regard différent paraît nécessaire à l'exploration des montagnes. On va trouver de nouvelles références dans les peintures de Salvator Rosa et dans les recherches qui ont inspiré l'art anglais des jardins à partir du début du XVIII ${ }^{*}$ siècle ${ }^{26}$.

Saussure et Wolf témoignent concrètement, dans leur travail d'observateurs et de descripteurs des Alpes, de la grande révolution épistémologique par laquelle s'achève le dépassement des théories classiques, autant sur le plan esthétique que sur celui de la métaphysique ou des conceptions scientifiques. A partir des années 1760 , comme d'autres voyageurs qui parcourent les Alpes,ils cherchent à expliquer la formation et l'état des montagnes en recourant aux théories récentes, et substituent aux formes de compréhension et aux croyances anciennes des attitudes qui répondent aux exigences nouvelles. Formulé très tôt dans la célèbre phrase d'Addison définissant le noble prospect des Alpes et l'agreable kind of horror qu'elles inspirent à qui les contemple 27 , le concept dusublime va servir à unifier la vision scientifique et le sentiment esthétique. Rappelons les différentes perspectives rassemblées dans ce concept.

L'usage classique du terme, tel que le définit Boileau dans sa traduction du texte grec attribué au rhéteur Longin, est principalement poétique. Il s'agit de décrire un langage qui excède les classifications rhétoriques, une manière de parler plus puissante que le style "élevé" assigné aux genres nobles. S’il parvient au Sublime, le poète produit chez son lecteur un choc émotif qui le soulève au-dessus de lui-même, au-dessus des contingences et des conventions ${ }^{28}$. Cet usage va perdurer durant le XVIII ${ }^{*}$ siècle ; il inspirera les théories qui chercheront à le dépasser,
24. Johiann-Jakob

Scheuchzer (1672.1733) a été membre de la Royal Sociely et a vécus longtemps à Londres. II y a publié en 1708 ses Itinera Alpina, somme des connaissances sur les Alpes dans tous les domaines.

25. Saussure ne rend jamais ridicules les légendes populaires qu'il rapporte; il adopte une attitude qu' on pourrait dire pré-ethnologique, en s'efforçant de les expliquer comme des

approximations de la vérité scientifique. 26. Sur cette question, la bibliographie est riche.

Voir notamment, pour les travaux récents parus en français, Michel Baridon, Nicolas Grimaldi et Alain Roger. Voir aussi la postface de Michel Conan à la réédition française de Gilpin. Trois Essais sur le beau pittoresque, Paris, 1982. 27. Les notes de voyage de Joseph Addison paraissent à Londres en 1705 /Remarks on several parts of italy... ) et sont traduites en français en 1722. Pour tous ces lextes, voir le Voyage en Suisse, à paraitre chez Laffont, coll. "Bouquins". 1995.

28. Le Sublime, dit Boileau dans sa Préface, est " cet extraordinaire ef ce merveilleux qui frappe dans le discours, et qui fait qu'un ouvrage enlève, ravit, transporte". Le Traité du Sublime est paru pour la première fois en 1674 
29. Voir les Lettres philosophiques (1734). Quinzième lettre, "Sur le système de l'attraction". Je cite d'après l'éd. Folio. Gallimard, 1986.

30. Ibid., Dix-septième lettre, "Sur l'infini et sur la chronologie».

31. Contemporain et compatriole de Saussure,

Marc-Théodore Bourrit apparut un temps comme son rival dans la découverte ef la description des itinéraires alpins de la vallée de

Chamonix. Il est loin pourtant d'avoir

l'importance scientifique et historique d'un Saussure.

32. A Philosophical

Enquiry into the Origin of our Ideas of the Sublime and Beautiful.

L'introduction de B. Saint

Girons à la traduction

française citée ci-dessus est tout à fait remarquable. c'est-à-dire à faire sortir la notion de sublime de la poétique pour lui donner de nouvelles extensions. On reconnaîtra en effet un sentiment sublime hors du champ du langage, dans la grandeur de certaines théories scientifiques et dans la beauté particulière de certains spectacles naturels. Lorsque Voltaire entreprend d'exposer aux lecteurs français le système de Newton, qu'il admire profondément, il parle de "sublimes idées ", de "sublime théorie", des " plus sublimes connaissances " 29 . C'est que pour lui Newton a donné aux hommes de son temps une juste vision de l'infini, en tant que le calcul peut l'expliquer, qu'une théorie peut en rendre compte. Le sublime apparaît dans le rapport entre la faiblesse de l'homme et l'immensité incommensurable de l'univers, parce que la rationalité scientifique inverse les proportions de ce rapport, et que la petitesse humaine se transforme en grandeur de l'esprit. La théorie en somme s'approprie l'infini en inventant "l'art de nombrer et de mesurer avec exactitude ce dont on ne peut pas même concevoir l'existence "30. Ce sublime restera à la science; de la physique ou de la géométrie newtoniennes, il passera aux autres savoirs capables de formuler théoriquement leurs objets, par reconstruction et par prévision : l'histoire naturelle de Buffon ou la théorie de la Terre que Saussure cherche à construire ont pu être dites sublimes.

Sur le plan esthétique, la qualification de sublime est donnée à des objets naturels, ou reproduits par l'art, qui témoignent de la grandeur ou de la puissance des phénomènes. La montagne (comme la mer) est grande pourvoyeuse de ce sublime-là. Dans la première littérature alpestre, un Bourrit use et abuse de l'expression $^{31}$. L'attribution aux choses-mêmes, en particulier aux paysages, conduit à dresser un catalogue d'objets sublimes, et donc à figer une notion dont l'intérêt réside, au contraire, dans son caractère constamment excessif. Edmund Burke, dans un essai paru en $1757^{32}$, cherche à dépasser cette aporie en proposant une conception psychologique fondée sur une théorie des passions. Il définit comme sublime la jouissance esthétique ( $d e-$ light) qui dépasse toutes celles que procure le sentiment du beau ; elle naît d'une tension entre l'attrait et la terreur. Mais cette expérience, parfois brutale, doit se résoudre dans la contemplation esthétique : il n'y a sublime que lorsque la conscience esthétique permet de transformer en jouissance une menace restée potentielle; le sujet perçoit la possibilité de sa mort dans l'excès terrifiant du phénomène, en même temps que lui est donnée l'assurance de sa conservation. La théorie 
burkienne est reprise et considérablement élargie par Kant dans le chapitre sur le sublime qu'il insère dans sa Critique de la faculté de juger. Kant connaît toutes les réflexions qui le précèdent et les reprend pour les systématiser. Il a soin de distinguer le " sublime mathématique " de ce qu'il appelle le "sublime dynamique dans la nature ". Cette expression elle-même pourrait prêter à confusion : il ne s'agit précisément pas d'attribuer la qualité de sublime aux objets du monde naturel, qui ne sont pour lui que de pures forces, mais au jugement seul, dans la mesure où celui-ci constate d'une certaine façon son échec au moment où il répond à la plus grande exigence extérieure. Cet échec est pourtant la marque de la supériorité de l'esprit parce qu'il fait apparaître les Idées comme des représentations transcendantales. Il ne saurait être question de développer ici une problématique qui a suscité de nombreux et importants commentaires 33 . Je voulais seulement indiquer que les développements de Kant me paraissent particulièrement en accord avec les pages de Saussure que nous avons analysées, de même qu'avec l'art du paysage chez Wolf ${ }^{34}$.

On voit bien chez chacun le double mouvement du sublime, qui en même temps exalte et humilie l'esprit, lui découvrant sa faiblesse devant la nature et le renvoyant à sa dimension propre. Cette tension entre le regard et l'objet, on continuera de l'appeler sublime tout au long du XIX' siècle, jusqu'au ressassement et au truisme, - jusqu'aux aventures de Monsieur Perrichon au Mont-Blanc... Pourtant, la génération romantique ne maintiendra pas l'unité entre esthétique et science réalisée par le concept, qui apparaîtra comme une sorte de compromis, apte à tout signifier. Elle fera éclater la perception de la montagne en plusieurs regards différents : celui du peintre et de l'amateur de paysages (ce sera le pittoresque), celui du scientifique (qui recherchera la seule objectivité) et cet autre enfin, où le sujet se perd dans son objet, où ces catégories mêmes sont périmées dans un mouvement d'essence religieuse, une philosophie de l'infini, dont plusieurs écrivains de la montagne s'inspireront.
33. On renverra ici au dernier paru, le livre de J.-F. Lyotard, Leçons sur l'analytique du sublime, Paris, Galilée, 1991. 34. Kant, qui n'a jamais quitté Königsberg, avait lu Saussure : peut-être un de ses correspondants lui avait-il parlé de Wolf? Peutêtre avaitil vu ses gravures, très fréquemment reproduites? La vérification documentaire n'est pas indispensable. dès lors qu'on considère les textes.

Claude Reichler
Manuscrit reçu:
janvier 94
accepté : avril 94 